Check for updates

Cite this: Phys. Chem. Chem. Phys. 2021, 23, 13714

Received 9th March 2021, Accepted 5th June 2021

DOI: $10.1039 / d 1 c p 01058 a$

rsc.li/pccp

\title{
Unravelling the structures of sodiated $\beta$-cyclodextrin and its fragments $\dagger$
}

\author{
Jordan M. Rabus, $\ddagger^{a}$ Robert P. Pellegrinelli, $\ddagger^{b}$ Ali Hassan Abi Khodr, ${ }^{b}$ \\ Benjamin J. Bythell, (D) ${ }^{a}$ Thomas R. Rizzo (D) ${ }^{b}$ and Eduardo Carrascosa (D) $\ddagger^{\star b}$
}

\begin{abstract}
We present cryogenic infrared spectra of sodiated $\beta$-cyclodextrin $[\beta-\mathrm{CD}+\mathrm{Na}]^{+}$, a common cyclic oligosaccharide, and its main dissociation products upon collision-induced dissociation (CID). We characterize the parent ions using high-resolution ion mobility spectrometry and cryogenic infrared action spectroscopy, while the fragments are characterized by their mass and cryogenic infrared spectra. We observe sodium-cationized fragments that differ in mass by $162 \mathrm{u}$, corresponding to $B_{n} / Z_{m}$ ions. For the $\mathrm{m} / \mathrm{z} 347$ product ion, electronic structure calculations are consistent with formation of the lowest energy 2-ketone $B_{2}$ ion structure. For the $\mathrm{m} / \mathrm{z} 509$ product ion, both the calculated 2-ketone $\mathrm{B}_{3}$ and the $Z_{3}$ structures show similarities with the experimental spectrum. The theoretical structure most consistent with the spectrum of the $\mathrm{m} / \mathrm{z} 671$ ions is a slightly higher energy 2-ketone $\mathrm{B}_{4}$ structure. Overall, the data suggest a consistent formation mechanism for all the observed fragments.
\end{abstract}

\section{Introduction}

The synthesis and biomedical applications of large cyclic molecular systems have received growing attention, mainly due to their ability to bind selectively to substrates based on non-covalent interactions. ${ }^{1}$ Crown ethers are arguably the most popular and best investigated host molecules for complexing metal ions and are widely used in organic and supramolecular chemistry. ${ }^{2,3}$ Cyclic oligosaccharides occur both naturally and synthetically, and they share similar properties with crown ethers in being able to act as a host for metal cations, organometallic complexes, and biologically relevant molecules. ${ }^{4}$ Among the advantages of these compounds is their relative ease of derivatization, allowing for a suitable functional group to be attached to the cyclic oligosaccharide, which can alter their affinity to a certain enantiomer and thus make them good chiral selectors. This is of great interest in separation techniques such as liquid chromatography and capillary electrophoreses. ${ }^{5,6}$

\footnotetext{
${ }^{a}$ Department of Chemistry and Biochemistry, Ohio University, 391 Clippinger Laboratories, Athens, Ohio 45701, USA

${ }^{b}$ Laboratoire de Chimie Physique Moléculaire, École Polytechnique Fédérale de Lausanne, EPFL SB ISIC LCPM, Station 6, CH-1015 Lausanne, Switzerland. E-mail: eduardo.carrascosacasado@epfl.ch

$\dagger$ Electronic supplementary information (ESI) available: Cryogenic IR spectra of $\beta$-CD complexed to different group I metal cations; schematic representation of candidate fragmentation pathways; experimental and theoretical IR spectra of putative fragment structures, including optimized geometries; further details of electronic structure calculations. See DOI: 10.1039/d1cp01058a

\$ These authors contributed equally to this work.
}

The best-known cyclic oligosaccharides are cyclodextrins (CDs), which are produced enzymatically from starch and occur in three different configurations: $\alpha, \beta$, and $\gamma$, made up of 6,7 , and 8 D-glucopyranose units, respectively, and linked together by $\alpha-(1-4)$ glycosidic bonds. ${ }^{7}$ The non-toxicity and inexpensive synthesis of cyclodextrins has made them the molecules of choice for many applications including drug delivery, cosmetics, and food processing. ${ }^{8-11}$ Very recently, a modified $\beta$-CD has been shown to have broad spectrum anti-viral properties, including against herpes and Zika. ${ }^{12}$

The properties of cyclodextrins arise from their unique conical cylindrical structure, with the hydroxyls pointing outwards while the glycosidic oxygens and the hydrogens line the interior, ${ }^{13}$ leading to a hydrophobic zone inside the cylinder, and a hydrophilic zone on the exterior. This allows CDs to form complexes with a wide variety of compounds, ranging from nonpolar molecules that are captured in the hydrophobic cavity, to polar molecules and ions which preferentially interact with the outer polar surface. Because of this feature, cyclodextrins are water soluble and can be used to bring poorly soluble compounds into aqueous solution by enclosing them in their hydrophobic cavity. For $\beta-\mathrm{CD}$, the $\mathrm{C} 2$ and $\mathrm{C} 3$ hydroxyls form a complete hydrogen-bonded network on the wide rim, while the C6 hydroxyls form a separate network on the narrow rim, resulting in a rigid structure (Fig. 1). ${ }^{14}$

Complexation of CDs with guest molecules and ions can distort these structures. It is therefore important to identify these subtle structural details and determine how they affect the physicochemical and biological properties of these species. For this purpose, techniques are needed that can isolate, 

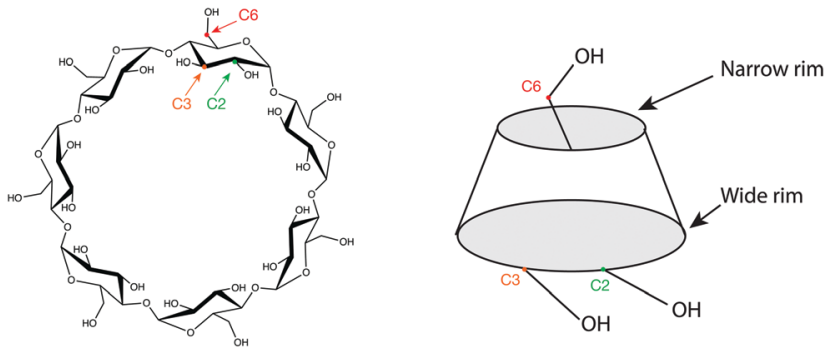

Fig. 1 Schematic representation of $\beta$-cyclodextrin, with its conical structure represented on the right. The carbon atoms connected to the hydroxyls are labelled C2, C3 and C6 for one glucose unit.

control and probe molecules. Tandem mass spectrometry $\left(\mathrm{MS}^{n}\right)$ coupled with soft-ionization techniques, such as electrospray ionization (ESI) and matrix-assisted laser desorption (MALDI), has emerged as a key tool to investigate large molecules and complexes. ${ }^{15}$ Mass spectrometry has thus been used to study many properties of cyclodextrins, including their host-guest interaction with various organic molecules, their binding selectivity with different metal cations, and their fragmentation patterns in the gas phase. ${ }^{16-21}$ MS coupled to collisioninduced dissociation (CID) is often used in biomedical and pharmaceutical contexts to reconstruct the primary structure and binding characteristics of complexed and functionalized CDs. For instance, fragmentation spectra can help determine the chirality of target analytes present in CD-based host-guest complexes ${ }^{22,23}$ as well as identify regioisomers of functionalized CDs. ${ }^{20}$ Furthermore, studies that use CDs to promote or catalyze the synthesis of biodegradable products rely on CIDMS studies to determine the number and structural arrangement of derivative host units attached to the CD guest, and consequently optimize the synthetic strategy. ${ }^{24,25}$ The homomolecular nature of cyclodextrins makes them interesting models for CID studies, because they are limited to three types of fragmentation: $\mathrm{B} / \mathrm{Y}, \mathrm{C} / \mathrm{Z}$ and cross-ring fragmentation. ${ }^{26}$ Fragmentation of $\beta$-CD-metal cation complexes by CID has primarily shown a series of product cations separated by $162 \mathrm{u}$, which corresponds to a dehydrated glucose unit that requires fragmentation of two glycosidic linkages. ${ }^{26-28}$ In addition, other cross-ring fragmentation channels have been observed, especially for anions. ${ }^{29}$

Despite being a powerful tool for fragmentation analysis, MS alone is unable to provide all desirable isomer-specific information, such as preferential isomer formation upon reaction or fragmentation. In the case of cationized cyclodextrins, MS studies have been unable to address whether CID preferentially forms $\mathrm{B}_{n}$ or $\mathrm{Z}_{m}$ ions. However, the addition of a vibrational spectroscopy dimension to MS can help identify structural details of CID fragments. While vibrational spectroscopy of ions in mass spectrometers dates back to early ' 80 's from the work of Beauchamp and coworkers, ${ }^{30,31}$ the development of user-friendly infrared lasers has resulted in the reemergence of infrared (IR) action spectroscopy as a structural probe for gasphase ions, including biomolecular ions. ${ }^{32-35}$ Our group has recently demonstrated that cryogenic IR spectroscopy coupled with ion mobility spectrometry (IMS) is able to identify various isomeric glycans ranging from monosaccharides to ninemembered N-glycans. ${ }^{36-39}$ In a very recent study we have been able to identify anomeric CID fragments from single glycans by their IR spectra. ${ }^{40}$ The advantage of measuring spectra of cryogenically cooled ions is that it greatly reduces thermal inhomogeneous broadening, resulting in significantly enhanced spectral resolution, which is particularly important for the analysis of large molecules possessing inherently congested spectra. ${ }^{33}$

Here we investigate the CID products of sodiated $\beta$-CD using cryogenic IR spectroscopy. Understanding which fragmentation pathway(s) is (are) responsible for product ions will provide insight into potential dissociation mechanisms of oligosaccharides which, until now, remain only partially understood.

\section{Methods}

\section{Experimental details}

To explore the fragmentation characteristics and corresponding structural signatures of $[\beta-\mathrm{CD}+\mathrm{Na}]^{+}$, tandem mass spectrometry, collision-induced dissociation and laser spectroscopy are combined in a homebuilt instrument, schematically depicted in Fig. 2, the details of which have been previously reported. ${ }^{41}$

$\beta$-Cyclodextrin (purchased from Sigma Aldrich and used with no further purification) was dissolved in a water-methanol $(1: 1)$ mixture at $0.1 \mathrm{mM}$ concentration, complexed to group I cations, and introduced into the gas phase via nanoelectrospray (nESI). In the case of sodiated $\beta$-CD cations, the electrosprayed ions were radially confined in an $\mathrm{RF}$ ion funnel at $\sim 1$ mbar before being accelerated through a potential difference of $\sim 240 \mathrm{~V}$ into a hexapole ion trap at a pressure of $10^{-2}$ mbar, creating fragments via CID. The trapped precursor

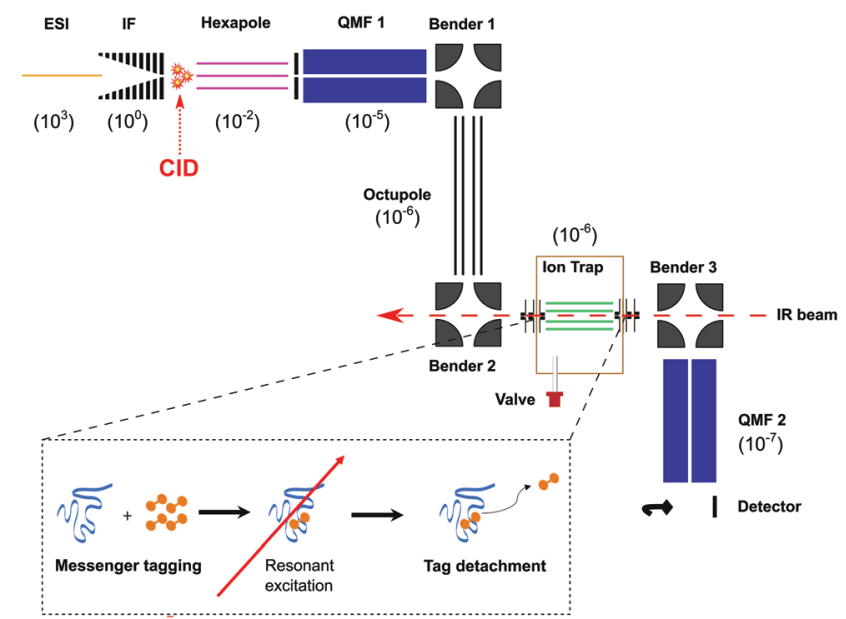

Fig. 2 Schematic representation of the experimental setup to obtain IR spectra of mass selected CID fragments under cryogenic conditions. Pressures, in mbar, are indicated in brackets for each differentially pumped section. The bottom left inset illustrates the principle of messenger tagging spectroscopy. 
ions and their fragments were then extracted as $100 \mu$ s pulses at $10 \mathrm{~Hz}$ and passed through a quadrupole mass filter, where ions of a designated $\mathrm{m} / \mathrm{z}$ range are selected. The transmitted ions were subsequently guided through two electrostatic benders and an octupole ion guide before being focused into a linear octupole ion trap that is cooled by a closed cycle cryocooler (SHI Cryogenics). Upon entering the trap, the ions are thermalized through collisions with a $\mathrm{He} / \mathrm{N}_{2}$ buffer gas mixture $(90: 10)$ that was previously introduced as $250-350 \mu$ s pulses and pre-cooled to the temperature of the copper trap housing. The trap temperature was held at 50-60 K using a resistive heater attached to the copper housing to avoid condensation of $\mathrm{N}_{2}$. To obtain single-photon infrared spectra at cryogenic conditions, the target ions were "tagged" by forming weakly bound complexes with $\mathrm{N}_{2}$. The trapped messenger-tagged species were then irradiated with IR light from a pulsed tunable optical parametric oscillator (OPO, LaserVision). In the event of resonant absorption, the vibrational energy rapidly redistributes within the molecule, thereby detaching the weakly-bound $\mathrm{N}_{2}$ tag. All the ions were then extracted from the trap and passed through a second quadrupole mass filter where they were detected using a Channeltron. By selecting the $\mathrm{m} / \mathrm{z}$ associated with the messenger-tagged target ion and monitoring its depletion as a function of laser wavenumber in a laser on-off experiment, cryogenic infrared action spectra were obtained. These spectra were normalized to the OPO power, which slightly varies over the wavelength range of the scan.

To investigate whether multiple isomers coexist in the gas phase for the studied species, analogous solutions of sodiated $\beta$-CD and $\alpha$-CD were electrosprayed into a recently developed ultrahigh-resolution ion mobility spectrometer attached to a cryogenic trap and a time-of-flight mass spectrometer. ${ }^{42}$ The ion mobility device allows for separation path lengths of over 10 meters and has demonstrated the ability to separate structurally analogous saccharide anomers. Thus, structural differences arising from differing hydrogen bonding arrangements, ring deformations (chair, boat, skew), and sites of sodiation should be well resolved.

\section{Computational approach}

To understand the formation mechanism and structure of the observed fragments, theoretical simulations were carried out on the three smallest fragment ions. As previously, ${ }^{43-46}$ simulations were performed using the genetic algorithm tool Fafoom $^{47,48}$ to enable effective characterization of the potential energy surface. The structures were optimized using the MMFF94 force field. ${ }^{49-53}$ This approach samples a wide range of ring structures incorporating multiple chair, boat, and skew forms, enabling a thorough interrogation of the potential energy surface. All oxygen sites had sodium cations added using custom scripts to generate starting points for the calculations. Geometry optimizations of the resulting candidate conformations were performed with the Gaussian 09 software package $^{54}$ at the HF/3-21G, B3LYP/6-31G(d), and B3LYP/ $6-31+G(d, p)$ levels of theory. ${ }^{55-57}$ For the putative charged fragments of the largest species with $m / z 671$, a combination of a preliminary PM6 optimization with a HF/3-21g single point energy calculation was performed prior to the aforementioned series of calculations. Degenerate structures were removed at each stage, and only the most competitive non-degenerate structures were optimized at each successively more realistic level of theory. Additional, targeted manual adjustment and supplementation of the structural pool analysed were performed to reduce the chance that chemically relevant species had been neglected. Subsequent simulations utilizing the CREST package, ${ }^{58-60}$ which systemically places a sodium ion at each lone pair of the molecule, with subsequent molecular dynamics using a semiempirical engine followed by B3LYP/6$31 \mathrm{G}(\mathrm{d})$ and B3LYP/6-31+G(d,p) calculations broadened the pool of low (and high) energy candidate structures but failed to generate new global minima. All minima were tested by vibrational analysis (all real frequencies). The potential energy surface generated combined the zero-point energy correction (ZPE) to the electronic energy $\left(E_{\mathrm{el}}, 0 \mathrm{~K}\right)$ for improved accuracy $\left(\Delta E_{\text {el+ZPE,0K }}\right)$. The related, standard enthalpy $\left(\Delta H_{298 \mathrm{~K}}\right)$, Gibbs free energy $\left(\Delta G_{298 \mathrm{~K}}\right)$, and entropy $\left(\Delta S_{298 \mathrm{~K}}\right)$ corrections to $298 \mathrm{~K}$ were also determined. Calculated B3LYP/6-31+(d,p) vibrational frequencies were utilized for comparisons with the experimental spectra. A scaling factor of 0.955 was used for all vibrational frequencies and a $5 \mathrm{~cm}^{-1}$ full width at half maximum Lorentzian line shape was employed for comparison to the experimental spectra. Targeted single point calculations at the M06-2X, and wb97X-D levels of theory were also performed on the selected minimum energy structures to help address variability. Subsequent targeted M06-2X and $\omega b 97 \mathrm{X}-\mathrm{D}$ optimizations and frequency calculations with $6-31+\mathrm{G}(\mathrm{d}, \mathrm{p})$ basis sets on $\mathrm{m} / \mathrm{z} 671$ candidates were consistent with the B3LYP data.

\section{Results and discussion}

\section{CID mass spectrum}

Fig. 3 presents mass spectra of sodiated $\beta$-CD under largely non-dissociative conditions (a) and CID conditions (b).

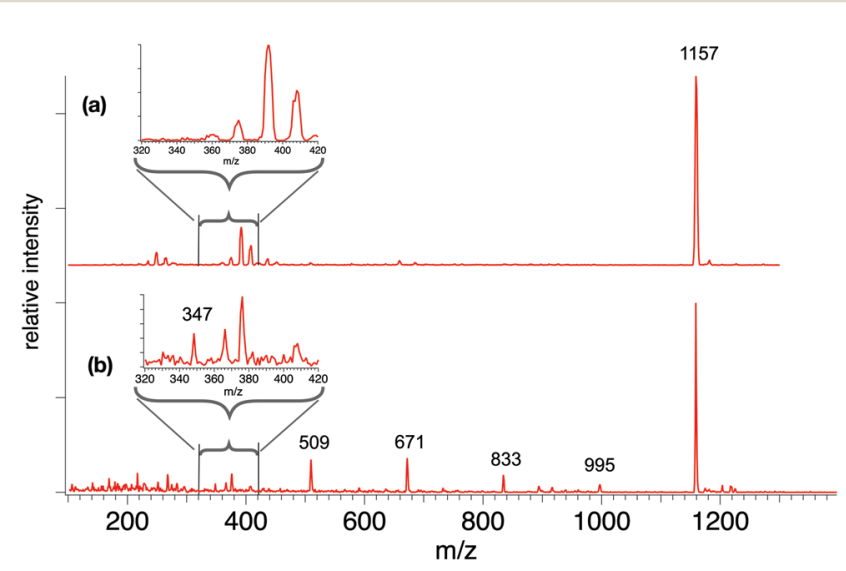

Fig. 3 CID-MS of sodiated $\beta-C D$ and its fragments, carried out with a potential difference of $100 \mathrm{~V}$ (a) and $240 \mathrm{~V}$ (b) in our tandem mass spectrometer. Consecutive loss of $162 \mathrm{~m} / \mathrm{z}$ is observed from the parent at $240 \mathrm{~V}$, while no fragments are observed at $100 \mathrm{~V}$. 
The spectrum in Fig. 3(b) exhibits the $[\beta-\mathrm{CD}+\mathrm{Na}]^{+}$precursor ion mass at $m / z 1157$, as well as a sequence of lower mass peaks at $m / z$ 995, 833, 671, 509 and 347.

This series indicates spacings of $162 \mathrm{u}$, a fragment moiety that has been previously observed ${ }^{20,27}$ and corresponds to a dehydrated glucose monosaccharide. Detection and analysis of the sodiated monomeric dehydrated glucose fragment at $\mathrm{m} / \mathrm{z} 185$ was not attempted, mainly due to the increased number of overlapping ions in the low mass range. A minor peak at $\mathrm{m} / \mathrm{z} 893$ corresponds to the loss of 264 mass units and is ascribed to a minor cross-ring fragmentation process also observed in previous investigations. ${ }^{27,29}$ The masses corresponding to the three- and four-glucose unit fragments $(\mathrm{m} / z 509$ and $\mathrm{m} / \mathrm{z}$ 671) appear as the most intense CID products, whereas formation of two- and six-residue fragments appear with much lower intensity.

It is difficult to determine whether the fragmentation occurs in a sequential or simultaneous manner. However, similar peaks and relative intensities are produced in a commercial time-of-flight (ToF) spectrometer (Waters Q-Tof Premier, Fig. S1, ESI $\dagger$ ) and our home-built instrument. To further investigate this, a series of CID experiments at increasing collision energies were performed on the Q-Tof, and all the main fragments appeared at the same energy threshold, providing no evidence for a sequential process (see Fig. S2, ESI $\dagger$ ).

\section{Ion mobility and cryogenic IR action spectra of sodiated $\alpha$-CD and $\beta-C D$}

Precursor $[\beta-\mathrm{CD}+\mathrm{Na}]^{+}$ions can adopt different conformations depending on the relative orientation of the $\mathrm{OH}$ groups at both the narrow and wide rims of the conical cylindrical structure (Fig. 1). The relative orientation of these donor-acceptor hydrogen bonding networks at both edges of the molecule can potentially lead to four different combinations, depending whether the hydrogen bonding pattern is clockwise $(\mathrm{cw})$ or counter-clockwise (cc): cc-cc, cc-cw, cw-cc and cw-cw. ${ }^{61}$ Distortion of these symmetric conformations through inclusion of $\mathrm{Na}^{+}$might significantly increase the number of conformers. To evaluate whether electrosprayed $[\beta-\mathrm{CD}+\mathrm{Na}]^{+}$ions form a distribution of conformers, additional experiments were carried out using a recently designed instrument that combines ultrahigh-resolution travelling-wave ion mobility spectrometry (IMS) using structures for lossless ion manipulation (SLIM) ${ }^{62,63}$ with cryogenic infrared spectroscopy to provide isomer-specific structural identification of complex molecules. ${ }^{42} \mathrm{~A}$ schematic representation of this instrument is shown in Fig. S3 (ESI $\dagger$ ). In this work, sodiated $\beta$-CD was cycled through the IMS section of this apparatus over a total length of $\sim 17$ meters, which has been shown to be sufficient to separate small structural differences. ${ }^{39}$ However as shown in Fig. 4(b), only one primary, well-resolved peak was observed in the arrival time distribution (ATD) under these conditions. A similar experiment on the six-member cyclic structure $[\alpha-\mathrm{CD}+\mathrm{Na}]^{+}$ also reveals a single peak in the ATD (Fig. 4(a)). These results can be potentially interpreted in three ways: (1) the monomodal ATDs demonstrate the presence of a single, stable gas-phase

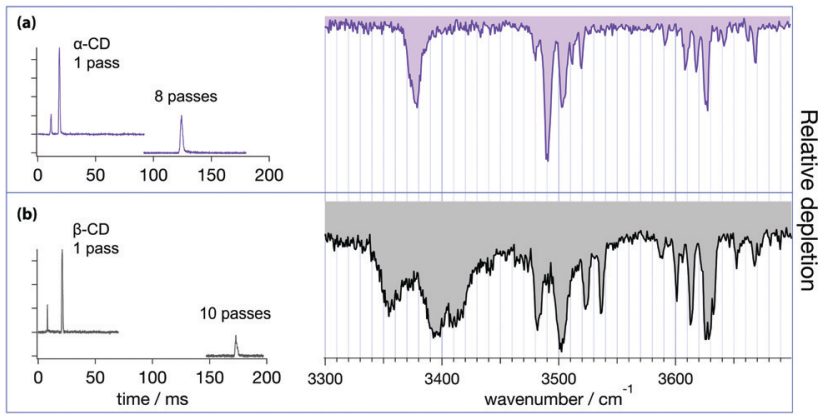

Fig. 4 Arrival times of $\left[\alpha-\mathrm{CD}+\mathrm{Na}^{+}(\mathrm{a})\right.$ and $[\beta-\mathrm{CD}+\mathrm{Na}]^{+}(\mathrm{b})$ and their corresponding cryogenic IR spectra. Note that the fast ATD peak in the left panels of (a) and (b) corresponds to doubly charged dimers of the corresponding cyclodextrins that cannot be discriminated by our quadrupole mass filter and are propelled faster through the IMS buffer gas due to their doubly charged character.

conformer for both sodiated $\alpha$ - and $\beta$-CD; (2) both cyclodextrins consist of multiple stable conformers (with different relative orientations of the hydrogen bonding networks, or different coordination number between the $\mathrm{Na}^{+}$and $\mathrm{CD}$, for example) that are so structurally similar that they cannot be separated using our SLIM-IMS device; or (3) the monomodal ATD results from multiple conformers that rapidly interconvert.

Option (3) appears highly unlikely, given the rigid geometry of both sodiated $\beta-\mathrm{CD}$ and $\alpha-\mathrm{CD}$ and the consequent significant strain associated with the concerted rearrangement of the hydrogen-bonded hydroxyl groups. We cannot completely rule out the possibility of (2), that structurally similar $[\alpha-\mathrm{CD}+\mathrm{Na}]^{+}$ or $[\beta-\mathrm{CD}+\mathrm{Na}]^{+}$conformers are present within the same ATD peak. Structural changes resulting from different coordination numbers of the sodium cation will likely lead to structural distortions easily resolved by our ion mobility device. Even if two or more cyclodextrin conformers would have such similar CCS that our SLIM-IMS is unable to resolve them, corresponding calculations would also not be able to reproduce the slight difference in CCS, as the resolution of our IMS instrument exceeds the accuracy of such calculations. As discussed more fully below, cryogenic IR spectroscopy is highly sensitive to structural differences, and the number of bands in each of the spectra of Fig. 4 is consistent with presence of single conformers. Thus, the most straightforward conclusion from the data of Fig. 4 is that both sodiated $\alpha$ - and $\beta$-CD exist as single conformers in the gas phase.

The cryogenic IR spectra corresponding to the monomodal ATD distributions of sodiated $\alpha$ - and $\beta$-CD shown in Fig. 4 were obtained by gating the monomer ATD peak, storing and tagging the corresponding ions in a cryogenic ion trap, and monitoring the depletion of tagged species as a function of IR wavenumber using a ToF spectrometer. Both IR spectra show similarities, with three distinct regions of IR transitions representing three types of $\mathrm{OH}$ oscillators. The lowest energy range of the spectra $\left(3330-3450 \mathrm{~cm}^{-1}\right)$ exhibits broad bands for both $[\alpha-\mathrm{CD}+\mathrm{Na}]^{+}$and $[\beta-\mathrm{CD}+\mathrm{Na}]^{+}$. The $[\alpha-\mathrm{CD}+$ $\mathrm{Na}]^{+}$spectrum presents a slightly asymmetric feature at $3375 \mathrm{~cm}^{-1}$, while the spectrum of $[\beta-\mathrm{CD}+\mathrm{Na}]^{+}$shows three distinct bands with maxima at 3355, 3395 and $3410 \mathrm{~cm}^{-1}$. Previous theoretical studies 


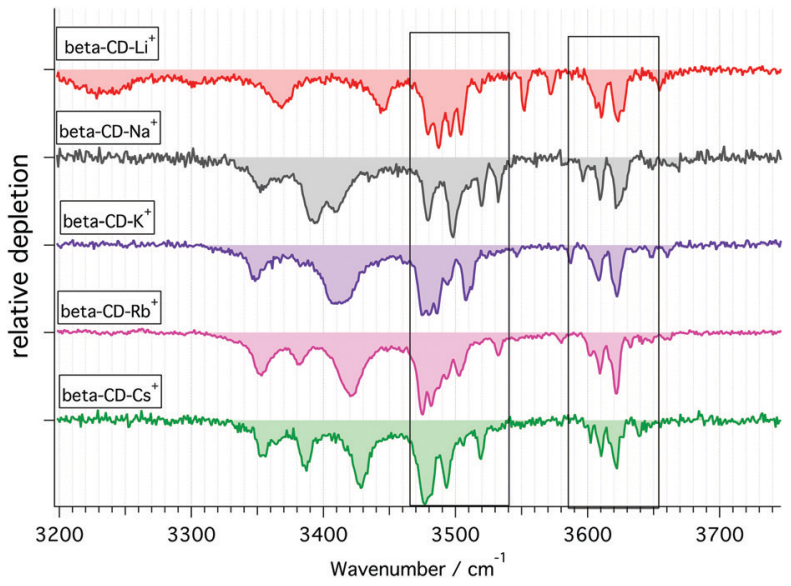

Fig. 5 Cryogenic IR spectra of $\beta-C D$ complexed with group I cations. The two marked areas denote the transitions associated to the $\mathrm{C} 2$ and $\mathrm{C} 3 \mathrm{OH}$ oscillators.

on neutral and metal-complexed CDs predict a tight hydrogen bonded network between the C6 hydroxyls on the narrow rim, and find that $\mathrm{Na}^{+}$and other metal cations preferentially coordinate with these hydroxyls. ${ }^{64-67}$

Additional cryogenic IR spectra of $\beta$-CD complexed to other group I metal cations (Fig. 5) demonstrate that the ionic radius strongly affects the bands below $3450 \mathrm{~cm}^{-1}$. The IR spectra in this wavenumber region show three major bands for all complexed $\beta$-CD cations, whereas only the complexes with $\mathrm{Cs}^{+}$and $\mathrm{Rb}^{+}$show significant spectral similarity. These experimental findings support the notion that the C6 hydroxyls are binding sites for the metal cations, but also hint to the formation of additional hydrogen bonding networks between these $\mathrm{C} 6 \mathrm{OHs}$, which vary in number and strength depending on the complexed metal cation. For instance, the small $\mathrm{Li}^{+}$will be complexed by a reduced amount of C6 O-atoms, thus likely promoting one or more strong hydrogen bonds between the hydroxyls undergoing metal complexation and other neighbouring free C6 OHs. Such a strong hydrogen bond could be the reason for the broad low energy band centred at $3225 \mathrm{~cm}^{-1}$, which is only present in the IR spectrum of $[\beta-\mathrm{CD}-\mathrm{Li}]^{+}$. In contrast to the lithium case, the large radii $\mathrm{Cs}^{+}$and $\mathrm{Rb}^{+}$cations will be complexed by many of the seven C6 hydroxyl groups of $\beta-\mathrm{CD}$, preventing the strong hydrogen bonds predicted for the $\mathrm{Li}^{+}$complex. These symmetry considerations agree with the theoretical predictions of Gamez and coworkers, which find a trivalent coordination of the C6 hydroxyls for the $[\alpha-\mathrm{CD}-\mathrm{Li}]^{+}$ complex, whereas the lowest energy $[\alpha-\mathrm{CD}-\mathrm{Cs}]^{+}$complex consists of five C6 Oxygens binding to the metal cation. ${ }^{67}$ Based on these arguments, an even stronger symmetric complexation could be inferred from the $[\alpha-\mathrm{CD}-\mathrm{Na}]^{+}$spectrum (Fig. 4(a)), where the presence of one single transition centred at $3370 \mathrm{~cm}^{-1}$ plausibly arises from a structural arrangement with the C6 hydroxyls binding to the metal cation, leading to a network of concerted hydrogen bonds. In contrast, the low energy spectral region in sodiated $\beta$-CD hints to a more asymmetric binding pattern to the metal cation.
The differences between sodiated $\alpha$ - and $\beta$-CD in this spectral region can be thus attributed to different sodium binding patterns and consequent symmetry distortions of the two molecules. In summary, we assign these low energy transitions to the C6 hydroxyl stretch vibrations and associate the differences in this region to the number of metal-binding $\mathrm{C} 6$ hydroxyls as well as the resulting strength of additional intramolecular hydrogen bonds between these OHs.

The higher energy part of the spectra shows two groups of narrow transitions in the $3450-3550 \mathrm{~cm}^{-1}$ and $3560-3700 \mathrm{~cm}^{-1}$ ranges. Based on the above assignment, these oscillators correspond to the $\mathrm{C} 2$ and $\mathrm{C} 3$ hydroxyls located on the wider rim. Theoretical studies have repeatedly shown that these hydroxyls form a hydrogen-bonded network that can adopt two different configurations around the rim ( $\mathrm{cw}$ and $\mathrm{ccw}$ ), either with the $\mathrm{C} 3$ hydroxyls hydrogen-bonded to the partially free $\mathrm{C} 2$ hydroxyls of the adjacent glucose moieties, or the reversed configuration. While these two possible configurations prevent us from assigning the groups of IR transitions to specific hydroxyls, the $3450-3550 \mathrm{~cm}^{-1}$ and $3560-3700 \mathrm{~cm}^{-1}$ transitions correspond to the hydrogen-bonded and partially free hydroxyls on the wide rim, respectively. The similarity between sodiated $\alpha$ - and $\beta$-CD in these spectral regions further supports that the metal cation does not bind here, but rather at the narrow rim.

\section{Cryogenic IR spectra of CID fragments}

Sodiated fragments of $[\beta-\mathrm{CD}+\mathrm{Na}]^{+}$were produced by collisioninduced dissociation between the ion funnel (IF, Fig. 2) and the hexapole followed by $\mathrm{m} / \mathrm{z}$ selection in the first quadrupole and storage in the cryogenic trap, where their IR spectra were recorded (Fig. 6(a-f)). These spectra provide well-resolved transitions that can be used to help determine the structure(s) of the fragment ions. Note that the IR spectrum of the precursor sodiated $\beta$-CD (Fig. 6(f)) obtained on the mass spectrometer

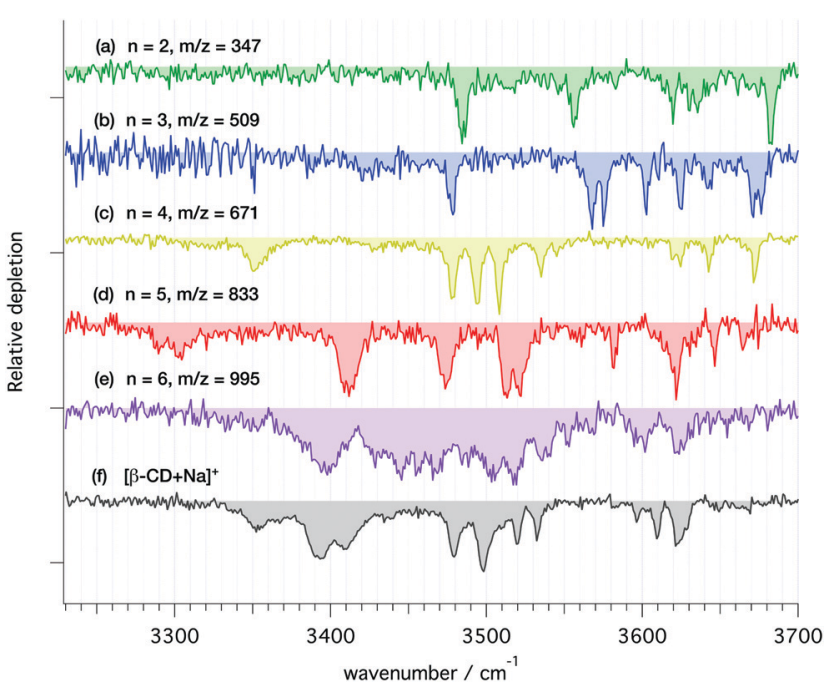

Fig. 6 Cryogenic IR spectra of the main CID fragments of $[\beta-\mathrm{CD}+\mathrm{Na}]^{+}$ $(a-e)$ and the parent ion (f). The index $n$ refers to the number of monosaccharides in each fragment. 
shown in Fig. 2 is highly consistent with the $[\beta-\mathrm{CD}+\mathrm{Na}]^{+}$ spectrum (Fig. 4(b)) obtained using the IMS-MS device (see Fig. S4, ESI $\dagger$ ). This spectral comparison demonstrates that the conformational landscape of sodiated $\beta-\mathrm{CD}$ is similar in both instruments. Following all above arguments, we expect that the CID fragments of Fig. 3(a) are produced from a single $[\beta-\mathrm{CD}+\mathrm{Na}]^{+}$conformer.

To associate the observed spectroscopic features with a specific structure, compositional sampling simulations followed by electronic structure and vibrational frequency calculations were performed on the sodiated fragments containing 2, 3, and 4 residues ( $\mathrm{m} / \mathrm{z} 347,509$, and 671, respectively).

The spectra of the 2 - and 3-residue sodiated fragments (Fig. 6(a and b)) exhibit a series of well-resolved bands above $3530 \mathrm{~cm}^{-1}$ as well as one band in the $3480-3490 \mathrm{~cm}^{-1}$ range. Neither spectrum show bands below $3470 \mathrm{~cm}^{-1}$. In both cases, the observed number of transitions match the expected number of $\mathrm{OH}$ stretch vibrations if one assumes that there is only a single conformer. The most distinct IR signatures are observed for the 4-residue fragment (Fig. 6(c)). The three clumps of wellresolved bands resemble the spectral features observed for $[\alpha-\mathrm{CD}-\mathrm{Na}]^{+}$(see Fig. 4(a)). Synthesis of similar cyclic tetrasaccharides has been previously carried out, ${ }^{68,69}$ and very recently the first synthesis of a 4-member cyclodextrin was reported. ${ }^{70}$ However, both the significant strain imposed on the glucose units $^{71}$ together with the predicted high barrier to re-cyclization make the formation of a 4-member cyclodextrin unlikely. Despite this, we tested this hypothesis computationally, as discussed below.

In contrast to the spectra of the smaller fragments, the 5- and 6-residue ions (Fig. 6(d and e)) exhibit a significant number of broad bands at lower wavenumber, which are characteristic of hydrogen-bonded $\mathrm{OH}$ stretch vibrations. The spectrum of the 5-residue fragment contains a few weak bands in the $3280-3320 \mathrm{~cm}^{-1}$ range, four strong broad features in the $3400-3550 \mathrm{~cm}^{-1}$ range, as well as several weaker transitions at higher wavenumber. There are fewer bands for this fragment than the number of $\mathrm{OH}$ oscillators, indicating that some bands overlap. The 6-residue fragment (Fig. 6(e)) exhibits a less resolved IR spectrum, with overlapping bands across the entire range between $3360-3630 \mathrm{~cm}^{-1}$. Comparing the $n=6$ fragment to the isomeric $[\alpha-\mathrm{CD}-\mathrm{Na}]^{+}$(Fig. 4(a)) reveals a difference in the number, position, and breadth of the bands, which may reflect the difference between a more symmetric, rigid, cyclic structure and an open, asymmetric one. The spectroscopic differences between the $n=6$ fragment and $[\alpha-\mathrm{CD}+\mathrm{Na}]^{+}$thus imply the presence of a substantial energy barrier to re-cyclization of the CID fragment. Rather, one (or several) open fragment structure(s) must be stabilized and kinetically trapped in its (their) potential minimum (minima) through collisional cooling.

\section{Comparison with theory}

Simulations of the 2-, 3-, and 4-residue fragments resulted in a significant number of low energy conformations. For the 2- and 3-residue fragments, simulations predict four distinct types of
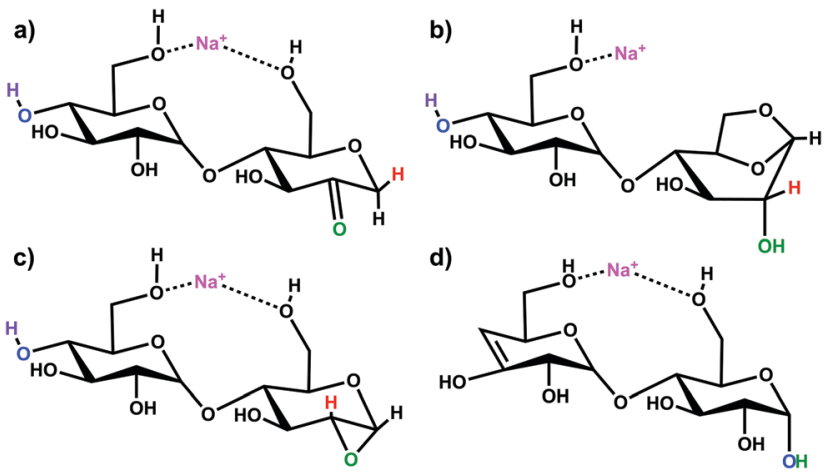

Scheme 1 Putative ion structure types potentially formed from [ $\beta-C D+$ $\mathrm{Na}]^{+}$, illustrated for the 2-residue, $\mathrm{m} / \mathrm{z} 347$ species: (a) 2-ketone $B_{2}$; (b) 1,6-anhydro $B_{2}$; (c) 1,2-anhydro $B_{2}$; and (d) $Z_{2}$ ion.

energetically stable structures (Scheme 1), with an additional cyclic form found for the 4-residue dissociation product.

Three of the four fragments are B ions, while the remaining fragment species is a product of a Z-type fragmentation. Our B3LYP data support 2-ketone B-type fragments ${ }^{43,44,72,73}$ as the lowest energy structures. The mechanistically less likely to be formed 1,6-anhydro B ion structure ${ }^{45,74}$ is next followed by the epoxide 1,2-andydro structures, which are consistently least energetically favourable $\left(\geq 85 \mathrm{~kJ} \mathrm{~mol}^{-1}\right)$. The $\mathrm{Z}$ fragments are predicted to be $10-20 \mathrm{~kJ} \mathrm{~mol}^{-1}$ higher in energy than the 2-ketone B ion structures. Large basis set M06-2X and $\omega \mathrm{b} 97 \mathrm{X}$ D single-point calculations predict the 2-ketone and 1, 6-anhydro terminated $\mathrm{B}$ ion structures and the $\mathrm{Z}$ ion structures to be of similar relative energy. All levels of theory substantially disfavour the strained 1,2-anhydro terminated B ion structures. The lowest energy structures of each type of fragment are shown in Fig. S5-S7 of the ESI, $\dagger$ and the corresponding relative energies are indicated in Table S1 (ESI $\dagger$ ).

For the 2-residue fragment $(\mathrm{m} / \mathrm{z} 347)$, Fig. 7 depicts the comparison between the experimental spectrum and the IR transitions calculated for the lowest energy $B_{2}$ and $Z_{2}$ ion structures. This result strongly suggests formation of a 2-ketone $\mathrm{B}_{2}$ ion structure (Fig. 6a). Consequently, we assign: (1) the $\sim 3485 \mathrm{~cm}^{-1}$ band to the symmetric $\mathrm{O}-\mathrm{H}$ stretch of the carbon 3 hydroxyl of residue 2, H-bonded to the adjacent ketone oxygen and the $\mathrm{O}-\mathrm{H}$ of the carbon 2 hydroxyl of residue 1 (also H-bonded); (2) the $3556 \mathrm{~cm}^{-1}$ band to the asymmetric stretch of the same hydroxyl groups; (3) the $3620 \mathrm{~cm}^{-1}$ band to the carbon 4 hydroxyl of residue 1, O-H stretch; (4) the $3630 \mathrm{~cm}^{-1}$ band to the carbon 3 hydroxyl of residue $1, \mathrm{O}-\mathrm{H}$ stretch; (5) the $3636 \mathrm{~cm}^{-1}$ band to the carbon 6 primary hydroxyl of residue 2, O-H stretch; and (6) the $3683 \mathrm{~cm}^{-1}$ band to the carbon 6 primary hydroxyl of residue 1 , free $\mathrm{O}-\mathrm{H}$ stretch. Following these assignments, and in contrast to the cyclic structures proposed for the metal complexes (Fig. 5), the low energy bands observed for the sodiated ring-opened $\beta$-CD fragments do not necessarily relate to the C6 hydroxyls.

The proposed mechanism for this fragmentation process is presented in scheme 2 and is adapted from earlier proposals for linear systems. ${ }^{43,44,72,73}$ Other candidate fragmentation 

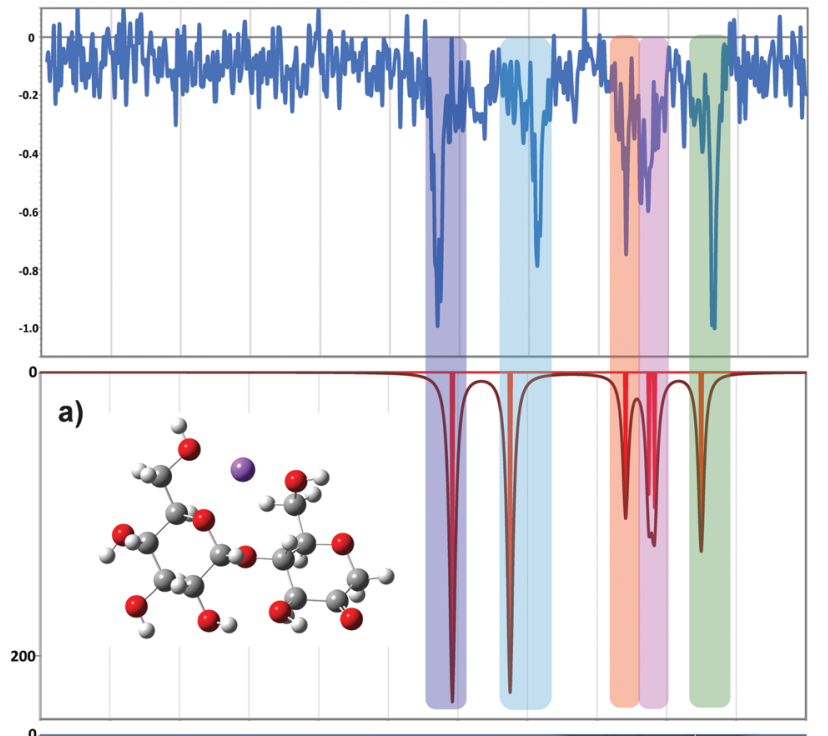

b)

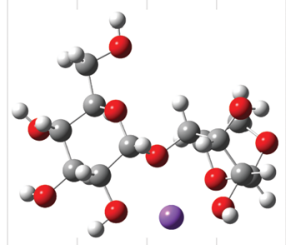

200

c)

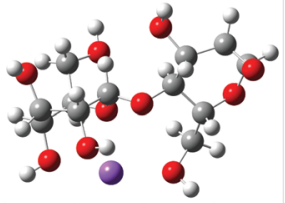

$200-$

d)

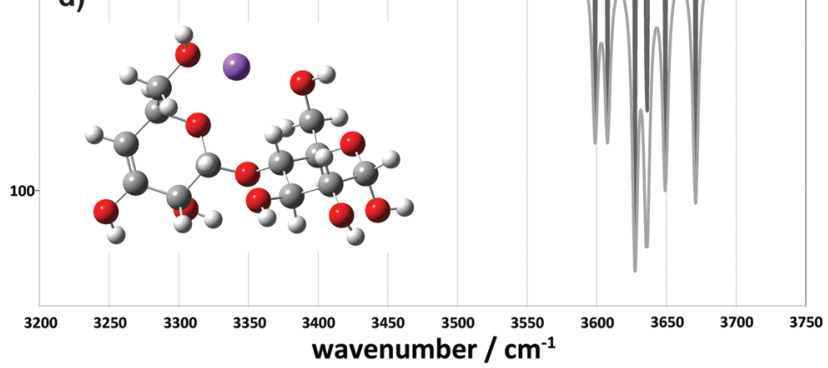

Fig. 7 Comparison between the experimental (top) and simulated IR spectra for the $m / z 347$ fragment. (a) Represents the ketone, (b) the 1-6 anhydro, (c) the 1-2 anhydro and (d) the $Z$ fragment structures.

pathways are detailed in Fig. S8 (ESI $\dagger$ ). The mechanism involves abstraction of a carbon 2 hydroxyl proton, formation of a ketone, and a 1,2-hydride shift with concerted glycosidic bond cleavage. ${ }^{43,44,72,73}$ This ring opening simultaneously creates the first 2-ketone and a new C4 hydroxyl group from the cleaved glycosidic linkage, i.e., nominally a linear $\mathrm{B}_{7}$ 2-ketone
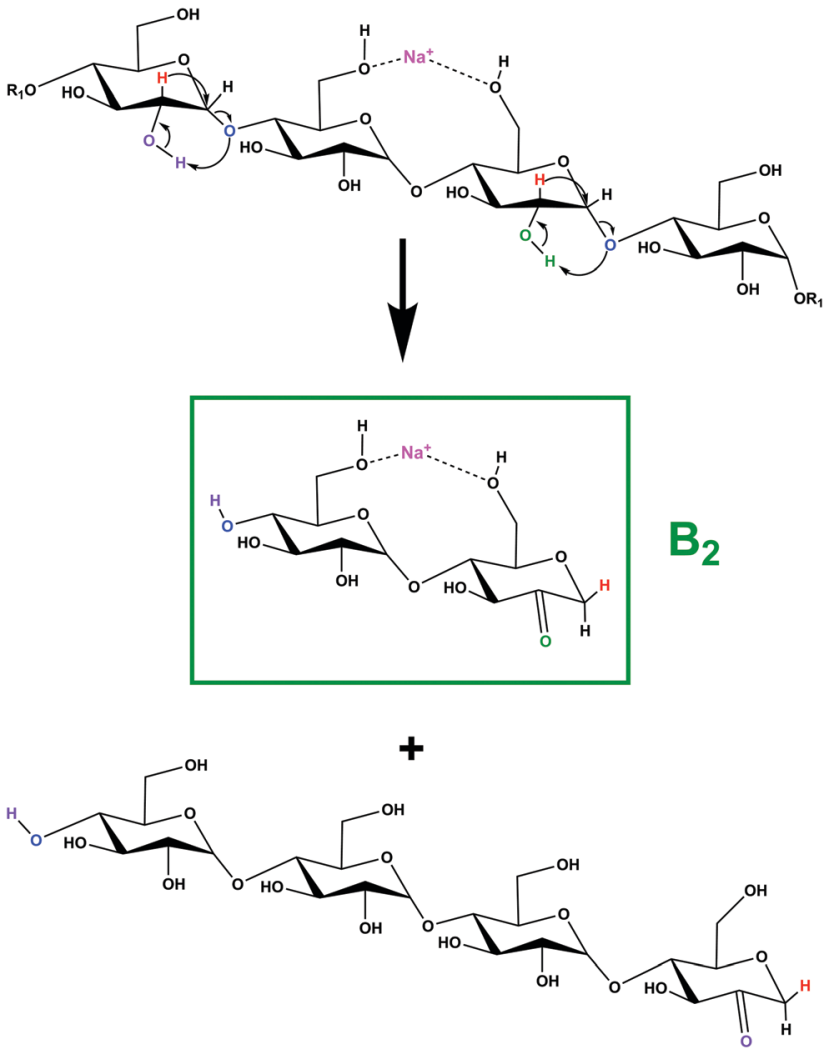

Scheme 2 Proposed fragmentation mechanism for the formation of the $\mathrm{m} / \mathrm{z} 347$ fragment.

terminated structure. Further dissociation of a second glycosidic bond in an identical manner results in generation of 2-residue and 5-residue structures with the sodium cation in an ion-molecule complex. ${ }^{74}$ Provided the complex has sufficient lifetime, a competition for the sodium cation ensues in which the fragment with the larger sodium affinity should most frequently predominate. ${ }^{45,74}$ If the 2-residue fragment separates with the sodium attached, an $\mathrm{m} / \mathrm{z} 347$ ion is detected, and if the 5-residue fragment separates with the sodium attached, an $m / z 833$ ion is detected.

Comparison between experimental and theoretical IR spectra of the 3-residue fragment is less conclusive (Fig. 8). None of the theoretical spectra of the lowest energy structures are perfectly consistent with experiment. The lowest energy 2-ketone type structure is reasonably consistent with experiment (Fig. 8a, mean deviation $=5.9 \mathrm{~cm}^{-1}, R^{2}=0.97$ ). However, the $\mathrm{Z}_{3}$-type structure shows a similar quality of fit (Fig. 8d, mean deviation $\left.=12.0 \mathrm{~cm}^{-1}, R^{2}=0.94\right)$. In contrast, the 1 , 2-anhydro $\mathrm{B}_{3}$ prediction lacks sufficient intermediate energy bands and there is no evidence to support any population of the 1,6-anhydro $B_{3}$ structure. The latter is consistent with structural arguments against the feasibility of the cross-ring proton transfers necessary to generate these structures from alpha linked sugars (Fig. S8c, ESI $\dagger$ ).

If our tentative assignment of the 2 -ketone $B_{3}$ or the $Z_{3}$ ion structure for the $m / z 509$ peak is correct, this offers a potential explanation for the origin of the $m / z 671$ spectrum-that is, 

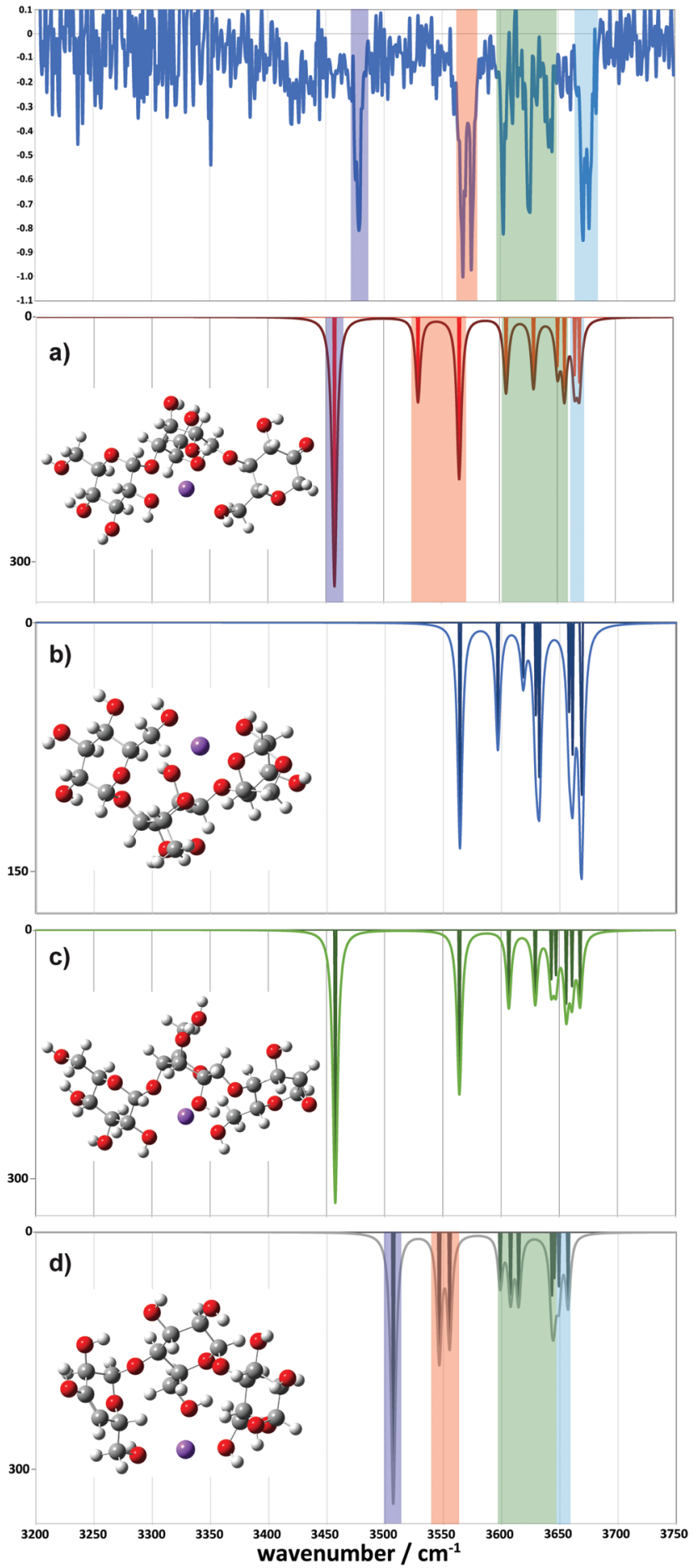

Fig. 8 Comparison between the experimental (top) and simulated IR spectra for the $m / z 509$ fragment. (a) represents the ketone, (b) the 1-6 anhydro, (c) the 1-2 anhydro $B_{3}$ and (d) the $Z_{3}$ fragment structures.

analogous 3-residue and 4-residue structures together with a sodium cation comprise the ion-molecule complex formed following dissociation of the 2 glycosidic bonds. As both neutrals are of similar size and comprise the same functional groups, similar sodium affinities should be expected, enabling both ions to be formed and detected, provided the ion-molecule complex

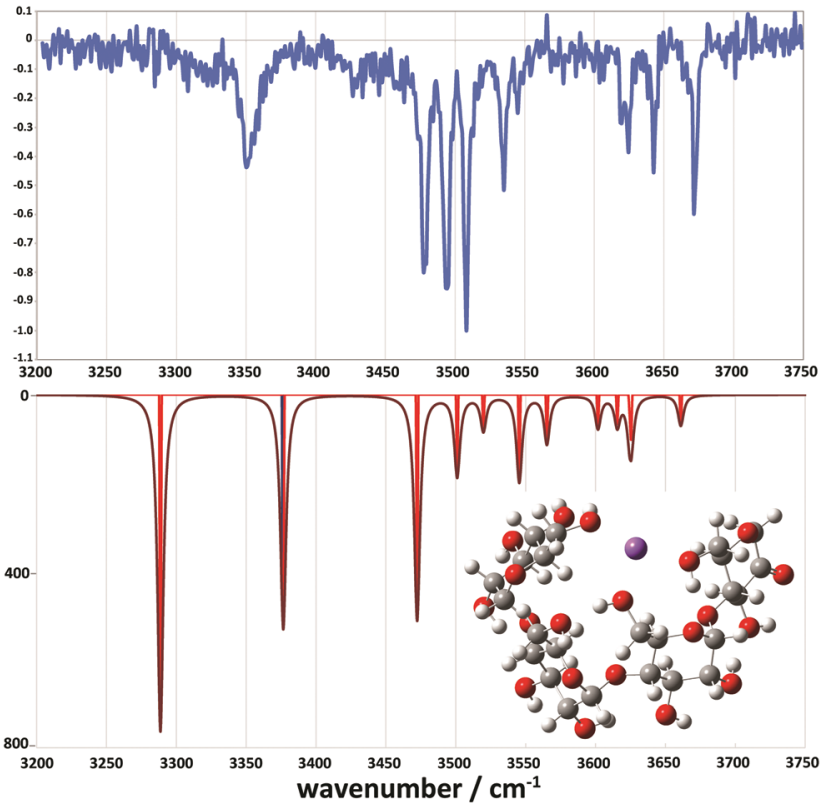

Fig. 9 Comparison between the experimental (top) and simulated IR spectra for the $\mathrm{m} / \mathrm{z} 671$ fragment. The simulated spectrum represents a 2-ketone $\mathrm{B}_{4}$ structure at $7.9 \mathrm{~kJ} \mathrm{~mol}^{-1}$.

has sufficient lifetime to enable sodium cation transfer between the fragments. ${ }^{45,74}$

Following this argument, either a 2-ketone $\mathrm{B}_{4}$ or a $\mathrm{Z}_{4}$ structure would be expected for the 4-residue fragment, but none of the lowest energy 2-ketone $\mathrm{B}_{4}$ nor $\mathrm{Z}_{4}$ theoretical spectra are particularly consistent with the experimental spectrum for $\mathrm{m} / \mathrm{z} 671$ (Fig. S7, ESI $\dagger$ ). This is similarly true for the other fragment structure types including potential cyclic candidate structures.

However, we did locate a low energy 2-ketone $\mathrm{B}_{4}$ structure that was far more consistent with the experimental spectrum (Fig. 9). The broad bands at low wavenumber correspond to strongly $\mathrm{H}-$ bonded $\mathrm{C} 6-\mathrm{OH}$ stretches (predicted at 3290 and $3377 \mathrm{~cm}^{-1}$ ), consistent with the broad feature at $\sim 3310-3380 \mathrm{~cm}^{-1}$. The bands at intermediate wavenumber correspond to a mixture of H-bonded environments ( $\mathrm{C} 3-\mathrm{OH}, \mathrm{C} 6-\mathrm{OH}, \mathrm{C} 2-\mathrm{OH}, \mathrm{C} 2-\mathrm{OH}$, $\mathrm{C} 2-\mathrm{OH})$ at $3473,3502,3520,3546$, and $3566 \mathrm{~cm}^{-1}$, respectively. The bands at high wavenumber $(3602,3616,3626$, and $3662 \mathrm{~cm}^{-1}$ ) comprise three $\mathrm{C} 3-\mathrm{OH}$ stretches and a free $\mathrm{C} 6-\mathrm{OH}$ stretch.

\section{Conclusions}

This work reports fingerprint vibrational spectra for a series of metal complexed $\beta$-CD cations, focusing on sodiated $\beta$-CD and its CID products. We obtain distinct, well-resolved infrared spectra for each of the main fragments. Simulations followed by electronic structure and vibrational frequency calculations were performed for the 2-, 3-, and 4-residue ions corresponding to $\mathrm{B}$ or $\mathrm{Z}$ ion structures and compared to the experimental results. The main findings of this work are: 
(1) Collisional activation of $[\beta-\mathrm{CD}+\mathrm{Na}]^{+}$results in a series of fragment ions differing by $162 \mathrm{u}$. Cryogenic IR spectroscopy experiments on the major fragment ions provide well-resolved vibrational fingerprints in the $3200-3750 \mathrm{~cm}^{-1}$ spectral region.

(2) Our combined experiments and calculations support the formation of a single gas-phase conformer in each of the $\mathrm{m} / \mathrm{z}$ 347,509 , and 671 species. The experimental spectrum for $n=5$ suggests formation of a single fragment conformer, while that for the 6-residue fragment suggests the presence of more than one stable conformer.

(3) There are four main types of potential fragmentation products for the 2-, 3-, and 4-residue fragments. For the 2residue fragment, the predicted lowest energy 2-ketone-type fragment is clearly the most similar to the experimental spectrum. For the 3-residue fragment, both the predicted lowest energy 2-ketone-type and $\mathrm{Z}_{3}$-type fragments show significant similarities to the experimental spectrum. The 4-residue fragment is also most consistent with a 2-ketone type structure, which in turn would support the same for the 3-residue fragment (Scheme 2). Thus, all the fragments seem to form $\mathrm{B}_{n}$ 2-ketone structures.

(4) The results presented here also demonstrate that cryogenic IR spectroscopy and theory can be used to directly relate spectroscopic patterns in CD-metal cationic complexes to the location and degree of coordination between the host CD and the metal ion.

In a broader context, this work demonstrates the potential of our method to unravel atomic-level structural features of complex biomolecular systems that can help address key macromolecular functions.

\section{Author contributions}

E. C. conceived the experiment; J. M. R. and B. J. B. performed the theoretical simulations; J. M. R. and B. J. B. analysed the simulation results; R. P. P. and E. C. carried out the experiments. R. P. P. analysed the experimental data; A. B. K. took the data shown in Fig. 4; J. M. R., A. B. K., B. J. B., and T. R. R. contributed to data discussion and interpretation; R. P. P., J. M. R., B. J. B., T. R. R. and E. C. wrote the manuscript.

\section{Conflicts of interest}

There are no conflicts to declare.

\section{Acknowledgements}

E. C., R. P. P., A. B. K., and T. R. R. thank the Swiss National Science Foundation (Grant 200020_184838), the European Research Council (Grant 788697-GLYCANAL), and the EPFL for the financial support of this work. J. M. R. and B. J. B. were supported by the National Science Foundation under CHE1948611 and ACI-1919789, Ohio University, and performed some calculations at the Ohio Supercomputer Center (http:// osc.edu/ark:/19495/f5s1ph73).

\section{References}

1 G. Gattuso, S. A. Nepogodiev and J. F. Stoddart, Chem. Rev., 1998, 98, 1919-1958.

2 C. J. Pedersen, J. Am. Chem. Soc., 1967, 89, 7017-7036.

3 C. J. Pedersen, J. Am. Chem. Soc., 1967, 89, 2495-2496.

4 F. Hapiot, S. Tilloy and E. Monflier, Chem. Rev., 2006, 106, 767-781.

5 A. F. Casy and A. D. Mercer, Magn. Reson. Chem., 1988, 26, 765-774.

6 P. Řezanka, K. Navrátilová, M. ̌̌ezanka, V. Král and D. Sýkora, Electrophoresis, 2014, 35, 2701-2721.

7 A. Biwer, G. Antranikian and E. Heinzle, Appl. Microbiol. Biotechnol., 2002, 59, 609-617.

8 G. Crini, Chem. Rev., 2014, 114, 10940-10975.

9 M. E. Davis and M. E. Brewster, Nat. Rev. Drug Discovery, 2004, 3, 1023-1035.

10 E. M. M. Del Valle, Process Biochem., 2004, 39, 1033-1046.

11 H. M. A. M. Dias, F. Berbicz, F. Pedrochi, M. L. Baesso and G. Matioli, Food Res. Int., 2010, 43, 1104-1110.

12 S. T. Jones, V. Cagno, M. Janeček, D. Ortiz, N. Gasilova, J. Piret, M. Gasbarri, D. A. Constant, Y. Han, L. Vuković, P. Král, L. Kaiser, S. Huang, S. Constant, K. Kirkegaard, G. Boivin, F. Stellacci and C. Tapparel, Sci. Adv., 2020, 6, eaax9318.

13 K. Lindner and W. Saenger, Angew. Chem., Int. Ed. Engl., 1978, 17, 694-695.

14 J. Szejtli, Chem. Rev., 1998, 98, 1743-1754.

15 J. Griffiths, Anal. Chem., 2008, 80, 5678-5683.

16 V. Gabelica, N. Galic and E. De Pauw, J. Am. Soc. Mass Spectrom., 2002, 13, 946-953.

17 C. B. Lebrilla, Acc. Chem. Res., 2001, 34, 653-661.

18 N. Marangoci, M. Mares, M. Silion, A. Fifere, C. Varganici, A. Nicolescu, C. Deleanu, A. Coroaba, M. Pinteala and B. C. Simionescu, Results Pharma Sci., 2011, 1, 27-37.

19 S. Reale, E. Teixidò and F. de Angelis, Ann. Chim., 2005, 95, 375-381. 20 S. Sforza, G. Galaverna, R. Corradini, A. Dossena and R. Marchelli, J. Am. Soc. Mass Spectrom., 2003, 14, 124-135. 21 P. Su, A. J. Smith, J. Warneke and J. Laskin, J. Am. Soc. Mass Spectrom., 2019, 30, 1934-1945.

22 F. He, J. Ramirez and C. B. Lebrilla, J. Am. Chem. Soc., 1999, 121, 4726-4727.

23 C. Sonnendecker, S. Thürmann, C. Przybylski, F. D. Zitzmann, N. Heinke, Y. Krauke, K. Monks, A. A. Robitzki, D. Belder and W. Zimmermann, Angew. Chem., Int. Ed., 2019, 58, 6411-6414. 24 C. Peptu, M. Danchenko, Ľ. Škultéty and J. Mosnáček, Molecules, 2018, 23(9), 2259.

25 Q. Zhang, G.-Z. Li, C. R. Becer and D. M. Haddleton, Chem. Commun., 2012, 48, 8063-8065.

26 B. Domon and C. E. Costello, Glycoconjugate J., 1988, 5, 397-409. 27 R. Frański, B. Gierczyk, G. Schroeder, S. Beck, A. Springer and M. Linscheid, Carbohydr. Res., 2005, 340, 1567-1572.

28 K. P. Madhusudanan, J. Mass Spectrom., 2003, 38, 409-416. 29 Y. Liu and D. E. Clemmer, Anal. Chem., 1997, 69, 2504-2509. 30 M. F. Jarrold, A. J. Illes, N. J. Kirchner, W. Wagnerredeker, M. T. Bowers, M. L. Mandich and J. L. Beauchamp, J. Phys. Chem., 1983, 87, 2213-2221. 
31 C. A. Wight and J. L. Beauchamp, J. Am. Chem. Soc., 1981, 103, 6499-6501.

32 J. Lemaire, P. Boissel, M. Heninger, G. Mauclaire, G. Bellec, H. Mestdagh, A. Simon, S. L. Caer, J. M. Ortega, F. Glotin and P. Maitre, Phys. Rev. Lett., 2002, 89, 273002.

33 T. Rizzo and O. Boyarkin, in Gas-Phase IR Spectroscopy and Structure of Biological Molecules, ed. A. M. Rijs and J. Oomens, Springer International Publishing, Switzerland, 2015, ch. 579, vol. 364, pp. 43-97.

34 T. R. Rizzo, J. A. Stearns and O. V. Boyarkin, Int. Rev. Phys. Chem., 2009, 28, 481-515.

35 Gas-Phase IR Spectroscopy and Structure of Biological Molecules, ed. A. M. Rijs and J. Oomens, Springer International Publishing, 2015.

36 I. Dyukova, E. Carrascosa, R. P. Pellegrinelli and T. R. Rizzo, Anal. Chem., 2020, 92, 1658-1662.

37 N. Khanal, C. Masellis, M. Z. Kamrath, D. E. Clemmer and T. R. Rizzo, Analyst, 2018, 143, 1846-1852.

38 C. Masellis, N. Khanal, M. Z. Kamrath, D. E. Clemmer and T. R. Rizzo, J. Am. Soc. Mass Spectrom., 2017, 28, 2217-2222.

39 S. Warnke, A. Ben Faleh, V. Scutelnic and T. R. Rizzo, J. Am. Soc. Mass Spectrom., 2019, 30, 2204-2211.

40 R. P. Pellegrinelli, L. Yue, E. Carrascosa, S. Warnke, A. Ben Faleh and T. R. Rizzo, J. Am. Chem. Soc., 2020, 142, 5948-5951.

41 A. Svendsen, U. J. Lorenz, O. V. Boyarkin and T. R. Rizzo, Rev. Sci. Instrum., 2010, 81, 073107.

42 A. Ben Faleh, S. Warnke and T. R. Rizzo, Anal. Chem., 2019, 91, 4876-4882.

43 M. T. Abutokaikah, J. W. Frye, J. Tschampel, J. M. Rabus and B. J. Bythell, J. Am. Soc. Mass Spectrom., 2018, 29, 1627-1637.

44 B. J. Bythell, J. M. Rabus, A. R. Wagoner, M. T. Abutokaikah and P. Maître, J. Am. Soc. Mass Spectrom., 2018, 29, 2380-2393.

45 J. M. Rabus, M. T. Abutokaikah, R. T. Ross and B. J. Bythell, Phys. Chem. Chem. Phys., 2017, 19, 25643-25652.

46 J. M. Rabus, D. R. Simmons, P. Maître and B. J. Bythell, Phys. Chem. Chem. Phys., 2018, 20, 27897-27909.

47 M. Marianski, A. Supady, T. Ingram, M. Schneider and C. Baldauf, J. Chem. Theory Comput., 2016, 12, 6157-6168.

48 A. Supady, V. Blum and C. Baldauf, J. Chem. Inf. Model., 2015, 55, 2338-2348.

49 T. A. Halgren, J. Comput. Chem., 1996, 17, 490-519.

50 T. A. Halgren, J. Comput. Chem., 1996, 17, 520-552.

51 T. A. Halgren, J. Comput. Chem., 1996, 17, 553-586.

52 T. A. Halgren, J. Comput. Chem., 1996, 17, 616-641.

53 T. A. Halgren and R. B. Nachbar, J. Comput. Chem., 1996, 17, 587-615.

54 M. J. Frisch, G. W. Trucks, H. B. Schlegel, G. E. Scuseria, M. A. Robb, J. R. Cheeseman, G. Scalmani, V. Barone, B. Mennucci, G. A. Petersson, H. Nakatsuji, M. Caricato, X. Li, H. P. Hratchian, A. F. Izmaylov, J. Bloino, G. Zheng, J. L. Sonnenberg, M. Hada, M. Ehara, K. Toyota, R. Fukuda, J. Hasegawa, M. Ishida, T. Nakajima, Y. Honda, O. Kitao, H. Nakai, T. Vreven, J. A. Montgomery Jr, J. E. Peralta, F. Ogliaro, M. Bearpark, J. J. Heyd, E. Brothers,
K. N. Kudin, V. N. Staroverov, R. Kobayashi, J. Normand, K. Raghavachari, A. Rendell, J. C. Burant, S. S. Iyengar, J. Tomasi, M. Cossi, N. Rega, J. M. Millam, M. Klene, J. E. Knox, J. B. Cross, V. Bakken, C. Adamo, J. Jaramillo, R. Gomperts, R. E. Stratmann, O. Yazyev, A. J. Austin, R. Cammi, C. Pomelli, J. W. Ochterski, R. L. Martin, K. Morokuma, V. G. Zakrzewski, G. A. Voth, P. Salvador, J. J. Dannenberg, S. Dapprich, A. D. Daniels, Ö. Farkas, J. B. Foresman, J. V. Ortiz, J. Cioslowski and D. J. Fox, Gaussian 09 Revision A.02, 2009.

55 A. D. Becke, J. Chem. Phys., 1993, 98, 5648-5652.

56 C. Lee, W. Yang and R. G. Parr, Phys. Rev. B: Condens. Matter Mater. Phys., 1988, 37, 785-789.

57 P. J. Stephens, F. J. Devlin, C. F. Chabalowski and M. J. Frisch, J. Phys. Chem., 1994, 98, 11623-11627.

58 S. Grimme, J. Chem. Theory Comput., 2019, 15, 2847-2862.

59 P. Pracht, F. Bohle and S. Grimme, Phys. Chem. Chem. Phys., 2020, 22, 7169-7192.

60 P. Pracht and S. Grimme, Chem. Sci., 2021, 12, 6551-6568.

61 W. Snor, E. Liedl, P. Weiss-Greiler, A. Karpfen, H. Viernstein and P. Wolschann, Chem. Phys. Lett., 2007, 441, 159-162.

62 L. Deng, Y. M. Ibrahim, A. M. Hamid, S. V. B. Garimella, I. K. Webb, X. Zheng, S. A. Prost, J. A. Sandoval, R. V. Norheim, G. A. Anderson, A. V. Tolmachev, E. S. Baker and R. D. Smith, Anal. Chem., 2016, 88, 8957-8964.

63 A. M. Hamid, S. V. B. Garimella, Y. M. Ibrahim, L. Deng, X. Zheng, I. K. Webb, G. A. Anderson, S. A. Prost, R. V. Norheim, A. V. Tolmachev, E. S. Baker and R. D. Smith, Anal. Chem., 2016, 88, 8949-8956.

64 C. Przybylski, V. Bonnet and C. Cézard, Phys. Chem. Chem. Phys., 2015, 17, 19288-19305.

65 A. Stachowicz, A. Styrcz, J. Korchowiec, A. Modaressi and M. Rogalski, Theor. Chem. Acc., 2011, 130, 939-953.

66 S. E. Angelova, V. K. Nikolova and T. M. Dudev, Phys. Chem. Chem. Phys., 2017, 19, 15129-15136.

67 F. Gámez, P. Hurtado, A. R. Hortal, B. Martínez-Haya, G. Berden and J. Oomens, ChemPhysChem, 2013, 14, 400-407.

68 G. C. Daskhan and N. Jayaraman, Chem. Commun., 2014, 50, 8554-8557.

69 K. Mukai, H. Watanabe, K. Oku, T. Nishimoto, M. Kubota, H. Chaen, S. Fukuda and M. Kurimoto, Carbohydr. Res., 2005, 340, 1469-1474.

70 D. Ikuta, Y. Hirata, S. Wakamori, H. Shimada, Y. Tomabechi, Y. Kawasaki, K. Ikeuchi, T. Hagimori, S. Matsumoto and H. Yamada, Science, 2019, 364, 674-677.

71 S. Immel, J. Brickmann and F. W. Lichtenthaler, Liebigs Ann., 1995, 1995, 929-942.

72 J.-L. Chen, H. S. Nguan, P.-J. Hsu, S.-T. Tsai, C. Y. Liew, J.-L. Kuo, W.-P. Hu and C.-K. Ni, Phys. Chem. Chem. Phys., 2017, 19, 15454-15462.

73 H. T. Huynh, H. T. Phan, P.-J. Hsu, J.-L. Chen, H. S. Nguan, S.-T. Tsai, T. Roongcharoen, C. Y. Liew, C.-K. Ni and J.-L. Kuo, Phys. Chem. Chem. Phys., 2018, 20, 19614-19624.

74 B. J. Bythell, M. T. Abutokaikah, A. R. Wagoner, S. Guan and J. M. Rabus, J. Am. Soc. Mass Spectrom., 2017, 28, 688-703. 